\title{
DIFFRACTION OF SH ELASTIC WAVES BY. A CRACK AND THE INTERCEPTION OF SOUND BY A RIGID WALL
}

\author{
Yasuo SATO \\ Earthquake Research Institute, University of Tokyo, Tokyo \\ (Received December 3, 1973)
}

\begin{abstract}
The propagation of SH elastic waves is studied assuming a surface source of disturbance. The medium is assumed to be a half space with a free crack which extends from the surface to a finite depth. The method is based on the numerical simulation using the finite difference technique, and the interception effect; or in other words the weakening of wave energy, by the crack is studied by the use of Fourier transform. If the correspondence between the SH wave field and that of the sound wave is considered, the same result is interpreted as the interception of the sound by a rigid wall, and that will be an important subject in big cities suffering from noise problems.
\end{abstract}

\section{Introduction}

The propagation of waves in an elastic half space when there is a crack or an obstacle in the medium is an interesting problem as well as important. The analytical method, however, requires high techniques in mathematics (ASMF Winter Annual Meeting, 1969. R. SAto's review (1967). See also N. YamaKawa (1962).) and even for the two dimensional case elliptic coordinates and Mathieu function, and other special functions are often necessary to provide a solution. Actual use of such a method, however, is fairly limited, because analytically soluble problems are not many, besides the nature of reflected and diffracted waves is difficult to grasp in spite of the complicated mathematical treatment. Consequently the experimental method, including the photoelastic technique, comes out to be a useful means for the elucidation of the phenomenon. The genius of Lord Rayleigh (see Theory of Sound) revealed the property pretty much, but such way of attack is not without weak points, and is somewhat far from our ideal to understand the nature of things thoroughly at a glance.

The present author has been trying to show the advantage of the numerical simulation by the use of high speed computers for a couple of years (SATô, $1972 \mathrm{a}, \mathrm{b})$. In the present paper also, an example of the simulation technique and the graphical representation thereof, are given for presenting some idea 
on the diffraction of $\mathrm{SH}$ waves by a free crack, which extends vertically from the free surface of elastic half space to a certain depth.

If the correspondence is recollected (SATô, 1959), which holds between the SH wave field and that of the sound wave, the solution given below gives a clue to the effect of the interception of noise by a rigid wall, and this will be an interesting subject since, nowadays, noises in big cities are becoming a serious problem everywhere.

\section{Numerical Methods}

From the viewpoint of technical aspect there is no difficulty in this problem. The free surface, including the crack, is without traction, that is to say (see Fig. 1)

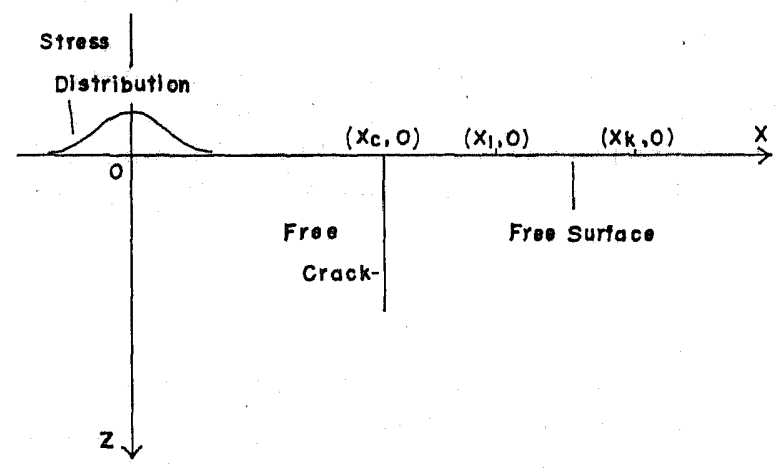

Fig. 1. Brief sketch of the model studied.

$$
\text { on the surface } \quad z=0 ; \quad \widehat{y z}=0
$$

and

$$
\text { on the crack } \quad x=x_{\mathrm{c}}, \quad 0<z<z_{\mathrm{c}} ; \quad \widehat{x y}=0
$$

which are equivalent to

$$
z=0 ; \quad v(x, \Delta z)-v(x,-\Delta z)=0
$$

and

$$
x=x_{\mathrm{c}}, \quad 0 \leq z \leq z_{\mathrm{c}} ; \quad v\left(x_{\mathrm{c}}+\Delta x / 2, z\right)-v\left(x_{\mathrm{c}}-\Delta x / 2, z\right)=0
$$

if the stress is expressed by the displacement $v$. In order to satisfy these boundary conditions, conventional formula of finite difference method is adopted (Alterman, 1968). The general wave equation is, as is well-known,

$$
\nabla^{2} v=\frac{1}{c^{2}} \frac{\partial^{2} v}{\partial t^{2}}
$$


or in the finite difference scheme

$$
\begin{aligned}
& \frac{1}{\Delta x^{2}}\{v(x+\Delta x, z)+v(x-\Delta x, z)-2 v(x, z)\} \\
& +\frac{1}{\Delta z^{2}}\{v(x, z+\Delta z)+v(x, z-\Delta z)-2 v(x, z)\} \\
& =\frac{1}{c^{2} \Delta t^{2}}\left\{v(x, z)_{t-\Delta t}-2 v(x, z)_{t}+v(x, z)_{t+\Delta t}\right\} .
\end{aligned}
$$

For the economy of the computer time and expense, $\Delta t$ is desirable to be taken as large as possible, but the stability condition for the numerical iteration gives the limit to it. The numerical data used in the present study is:

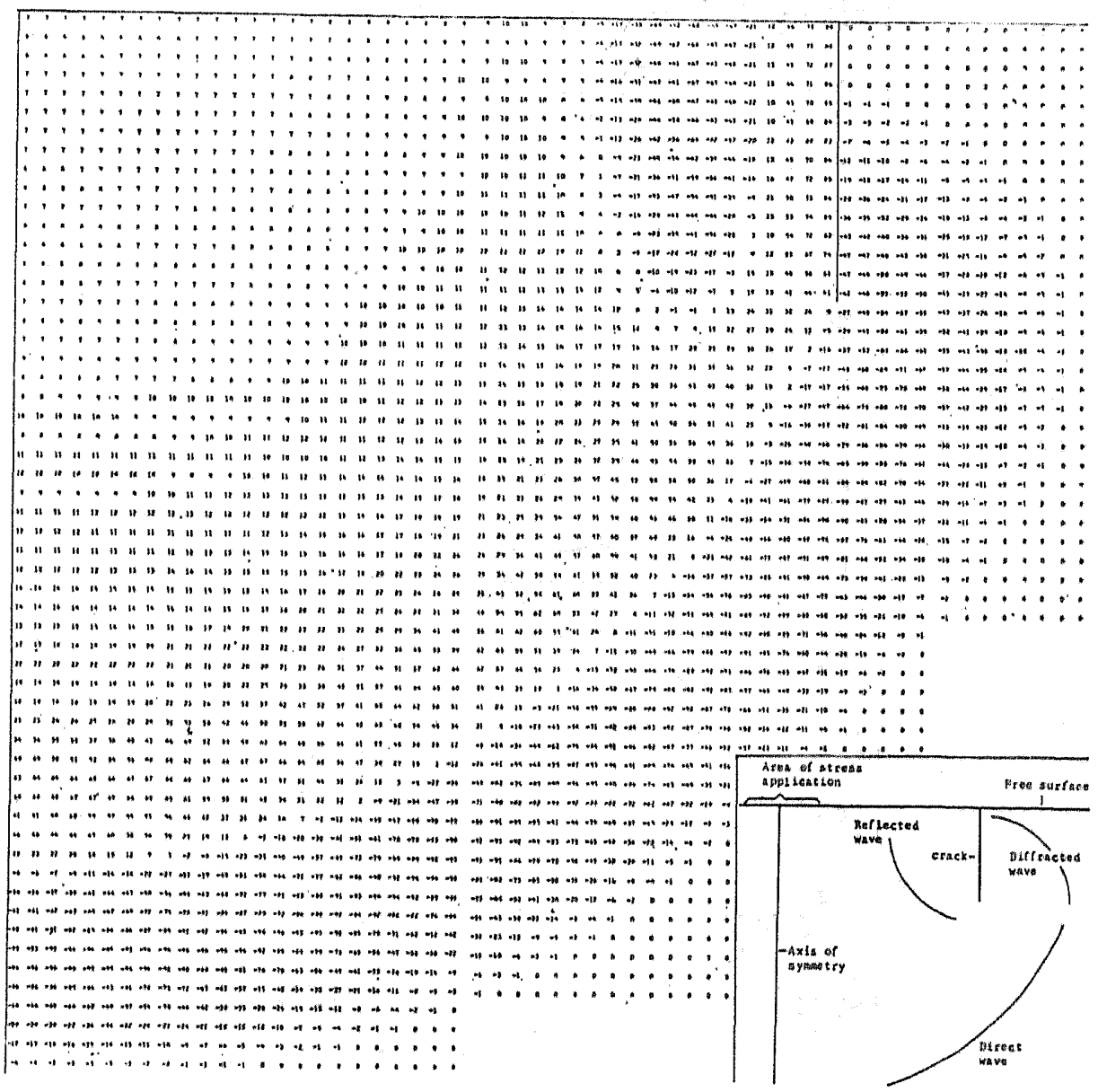

Fig. 2. SH wave displacement field. The numerals at the grid points are proportional to the displacement. The direct wave, reflected wave at the crack and the diffracted wave propagated into the shadow zone are observed. Time $=79 \mathrm{\Delta t}$, which is the moment just before the arrival of the diffracted wave at the free surface. 
The source function $=F(t, x)=f(t) \cdot g(x)$

$$
\begin{aligned}
& f(k \Delta t)= \begin{cases}\sin (2 \pi k / n), & 0 \leqq k \leqq n \\
0 & k<0, \quad k>n\end{cases} \\
& g(k \Delta x)= \begin{cases}{[1+\cos (\pi k / m)] / 2} & |k| \leqq m \\
0 & |k|>m\end{cases}
\end{aligned}
$$

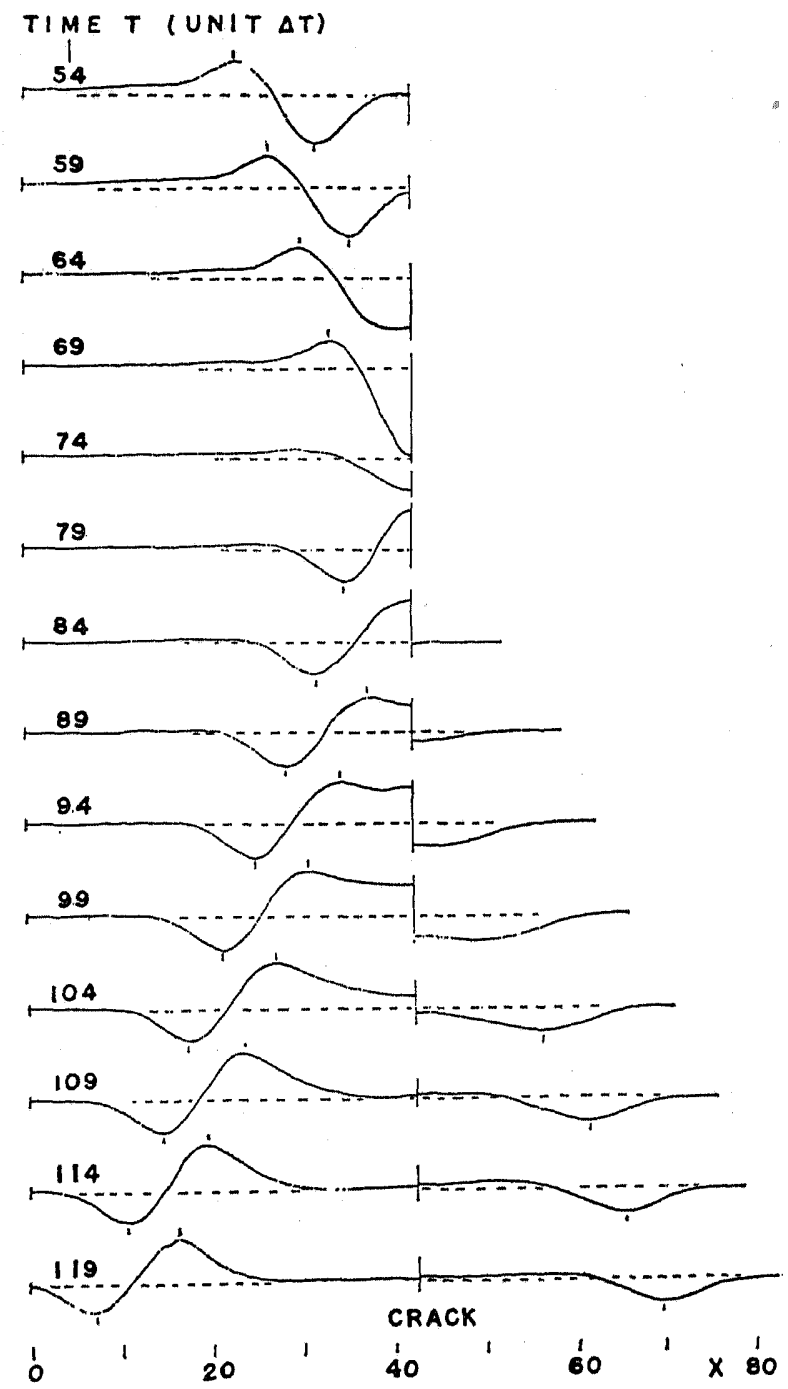

Fig. 3 a. Wave profiles on the free surface. The discontinuity of displacement on both sides of the crack is observed. Unit of $x$ is $\Delta x . x_{\mathrm{c}}=42.5 \Delta x, z_{\mathrm{c}}=14 \Delta z$. (See the numerical values assumed in (4).) 


$$
\begin{aligned}
& \text { Velocity }=\mathrm{c}=1.0 \\
& \text { Space interval }=\Delta x=\Delta z=0.2 \\
& \text { Time interval }=\Delta t=0.14 \\
& \text { Crack location }=x_{\mathrm{c}}=42.5 \mathrm{Ax} \\
& \qquad z_{\mathrm{c}}=14 \mathrm{\Delta z}
\end{aligned}
$$

and $n=24, m=4$.

Since the SH wave has the displacement oriented in the direction perpendicular to the $x-z$ plane, which is the plane of wave propagation, no vector representation is required for the wave field, but scalar values are enough, so a line printer output is enough to express the result, and an example is given in Fig. 2. The wave profiles for a number of time stages (Fig. 3a) show brief feature of the propagation of waves, including the reflection from the crack and the diffraction into the shadow zone.

\section{Fourier Analysis}

The above figures might help us to get a general idea of the diffraction pattern, but the dependence of the diffraction effect by the crack on the wavelength or period is by no means clear from these figures. One solution for this problem is to assume a simple harmonic source function instead of an aperiodic type employed in the expression (4). Another remedy is the application of the Fourier transform, because the source function here used is, as the Fourier's theorem teaches us, a kind of superposed form of various simple harmonic oscillations, and so is the diffracted disturbance observed behind the crack. Hence the comparison of the spectrum of the direct waves and that of the diffracted ones indicates the effect of interception. The calculated displacements as the function of time on the surface for the points in front of, as well as behind the crack, are shown in Fig. $3 \mathrm{~b}$ together with the expected direct wave when the crack does not exist. The spectrum amplitude (Fig. 4) certainly becomes smaller at the backside of the crack, which fact is easily expected even from the original disturbance shown in the figure. Using the result just obtained, more detailed study could be done quantitatively, for example the travel time, the velocity, the dependence on wavelength and the azimuthal characteristics of the diffracted waves.

In the calculation here carried out, the center of the source is situated at the distance $x_{c}$ from the crack, while the observation points are at $x=$ $x_{1}, x_{2} x_{3}, \ldots$ that means at the distance $x_{1}-x_{c}, x_{2}-x_{c}, x_{3}-x_{c}, \ldots$ from the crack. In this kind of field problem, however, the reciprocal formula generally holds (MORSE and Feshbach, 1953; SATO, 1952), hence the source and the observation points might be interchanged so that the effect of different focal 


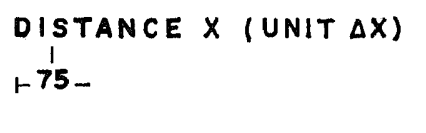

167-
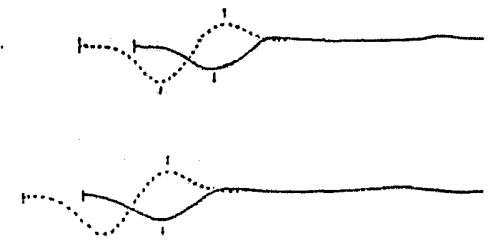

$159-$

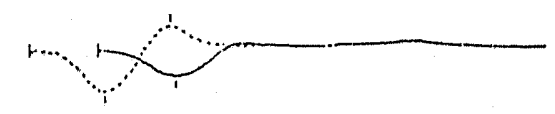

$+51-$
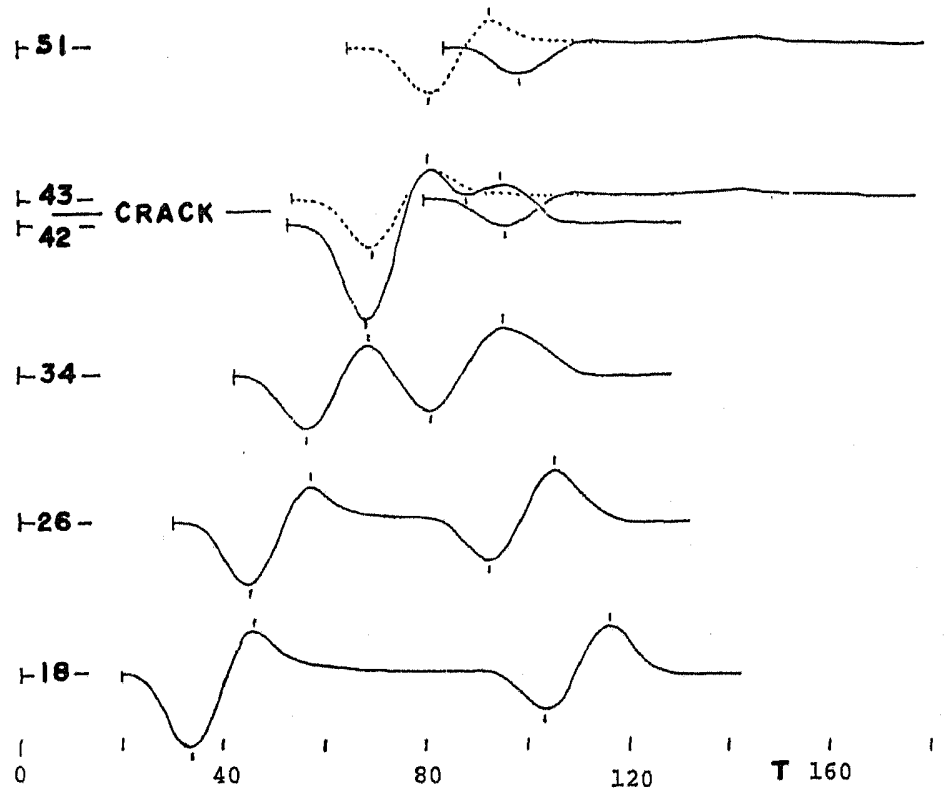

Fig. 3b. Displacement as a function of time at a number of points on the surface (solid line). The broken line is the expected displacement when there is no crack. The comparison with the solid line indicates the interception effect of the crack. In front of the crack, the reflected waves are observed. Unit of time $t$ is $\Delta t$. $(\Delta t=0.14, \Delta x=0.2)$

and crack distances can be inferred, from the present result without trying calculations anew.

4. Interpretation of the SH Wave Calculation as the Sound Interception Effect

As was pointed out in the introduction, the SH wave problem can be interpreted as the sound wave propagation, as long as the correspondence given below is adopted, namely; 


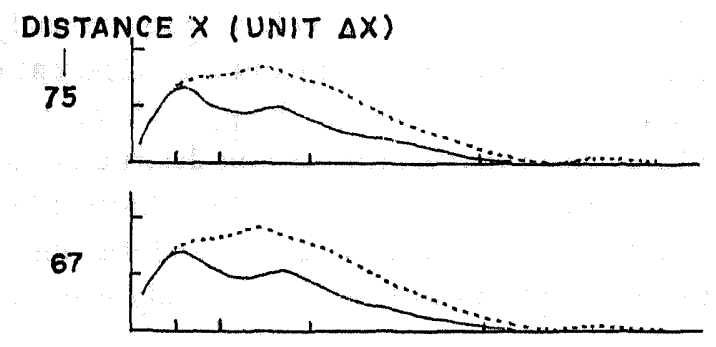

59

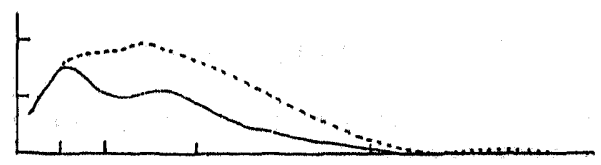

51

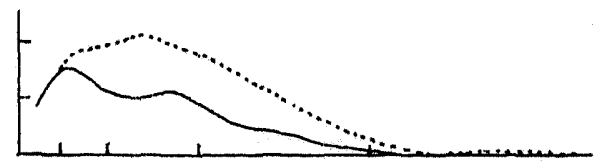

43

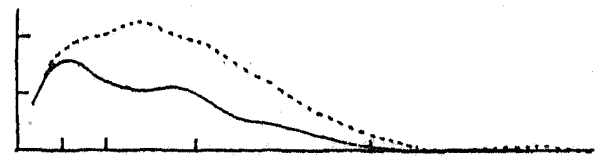

30

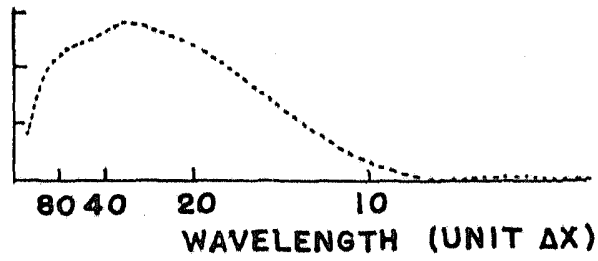

Fig. 4. Spectra of observed displacement (solid line), and that which is expected when no crack is assumed (broken line). The comparison of these curves gives the quantitative effect of the crack as the barrier of waves. (Crack length $=14 \Delta x$ )

\begin{tabular}{ll}
\multicolumn{1}{c}{ SH wave } & \multicolumn{1}{c}{ Sound wave } \\
Free surface & Rigid surface \\
Free crack & Rigid wall \\
Displacement & Sound pressure
\end{tabular}

Consequently, if the Fig. 1 is made up-side-down, the free surface to be a rigid ground and the crack is interpreted as the sound interception wall, the present solution can show the effect of the rigid wall as the sound barrier. Such a kind of problem is an important one in view of the seriousness of the noise disturbance for the inhabitants in big cities. For this problem, simple model experiments may yield useful result in a limited condition, but the present numerical simulation will probably prepare better and more versatile 
data since the parameters are changed in a wide range quite easily. Besides, more complicated models, say heterogeneous medium or different configuration of cracks and obstacles, can also be treated without much increasing the difficulty of calculation. The author believes, for the non-destructive testing of materials also, the numerical simulation by means of the finite difference technique will become a powerful tool in the near future, and starting from this kind of simple example the development of this field of study is desired.

\section{REFERENCES}

Alterman, Z. S., Finite difference solutions to geophysical problems, J. Phys. Earth, 16 (Special Issue), 113-128, 1968.

ASMF Winter Annual Meeting, Los Angeles, Calif., Wave propagation in solids, 1969.

Morse and Feshrach, Methods of Theoretical Physics, \$ 7.3, McGraw-Hill, New York, 1953.

RAYLEIGH, Lord, Theory of Sound II, 139-143.

SATo, R., Diffracted Waves, Zisin (ii), 20 (Special Issue), 74, 1967.

SATÔ, Y., Study on surface waves VI, Bull. Earthq. Res. Inst., 30, 101-120, 1952.

SATÔ, Y., Numerical integration of the equation of motion for surface waves in a medium with arbitrary variation of material constants, Bull. Seism. Soc. Amer., 49, 57-77, 1959.

SATÔ, Y., A small numerical experiment of the generation, propagation and the reflection, diffraction of elastic waves, Zisin (ii), 25, 189-190, 1972 a.

SATO, Y., A numerical experiment on wave propagation in an elastic quarter space, J. Phys. Earth, 20, 287-299, 1972 b.

Yamakawa, N., Scattering and attenuation of elastic waves I, II, Geophys. Mag., 31, 63-95, 97-103, 1962. 